\title{
Safe nutritional access in immunosuppressed transplant patients; Laparoscopic gastrostomy/jejunostomy feeding tube
}

\author{
Eric Hansen ${ }^{1}$, Conor O’Neill ${ }^{2}$, and Carlos E. Marroquin ${ }^{2 *}$ \\ ${ }^{1}$ Johns Hopkins School of Medicine, Baltimore, MD, USA \\ ${ }^{2}$ Larner College of Medicine at The University of Vermont Medical, Department of Surgery, Burlington VT, USA
}

\begin{abstract}
End organ disease is associated with sarcopenia and overall poor conditioning and malnutrition. As such, nutritional support is a critical component of post transplant recovery. Yet it is associated with multiple morbidities and is also associated with significant mortality. Morbidities can occur as a result of efforts to deliver sustenance with aspiration leading to pulmonary and infectious complications. Moreover, technical misadventures have been associated with peritonitis secondary to perforation, leaks and malpositioning of percutaneously placed feeding tubes.
\end{abstract}

Herein we describe a safe alternative to deliver critically necessary nutritional support with a laparoscopic assisted gastrostomy or jejunostomy feeding tube.

\section{Introduction}

The importance of maintaining adequate nutrition in the postoperative setting cannot be overstated. Enteral nutrition maintains the integrity of the intestinal mucosa decreasing bacterial translocation, and improving the overall health of the mucosal associated lymphoid tissue and immunity [1]. Patients who are malnourished are functionally immunosuppressed [2] increasing their susceptibility to post-operative infections. As such, enteral nutrition has been shown to decrease infectious complications [3]. Patients receiving immunosuppressive therapies following solid organ transplantation, those being treated with systemic chemotherapeutic agents for malignancies and patients who are functionally immunocompromised by chronic illness and/or a prolonged ICU course are at greatest risk for poor wound healing and compromised outcomes. Therefore, poor nutritional reserve should be cause for prudence before embarking upon any surgical procedure, no matter how mundane or simple it may seem. Moreover, being able to provide enteral nutritional support once a substantial surgical procedure has been performed will improve its outcome.

The discovery and development of parenteral nutrition is one of the greatest accomplishments of modern medicine. Nevertheless, the advantages of enteral feeding are profound [3]. Meeting this critical need in malnourished and deconditioned patients can often be challenging at best. These patients are often ventilator dependent with tracheostomies or endotracheal tubes and so profoundly deconditioned they lack the energy to consume sufficient calories orally to meet their metabolic demands. Given their profound weakness and poor coordination, they are at increased risk of aspiration when they are provided oral food stuff as they are unable to protect their airway. While feeding patients through nasogastric tubes has gained popularity, we continue to frown upon this practice as the risk of aspiration from gastric feeding [4] is not trivial and occurs with greater frequency than jejunal feeding. Using post-pyloric feeding tubes is a good temporary means of nutrition, but these frequently become dislodged or mal-positioned resulting in nutritional "holidays" affecting the ability to provide continuous nutrition to these critically ill patients. Finally, the presence of these devices in the oropharynx continue to affect pulmonary conditioning, are a source of nasopharyngeal infections and are barriers to nursing home and rehabilition placement. Finding access in patients who are unable to take anything by mouth has traditionally been accomplished by one of two means. Open gastrostomy/jejunostomy versus percutaneous endoscopic gastrostomy (PEG).

The open technique offers the advantage of placement of the feeding tube into the GI tract under direct visualization. Since patients that are both immunosuppressed and severely malnourished do not wall off collections or form fibrous tracts as readily as their immunocompetent counterparts, the ability to anchor the gastric or jejunal wall to the anterior abdominal wall offers greater safety than the percutaneous techniques. However, this requires an incision. An open incision, even if limited to $5 \mathrm{~cm}$, is less than ideal in an immunosuppressed and malnourished patient with impaired wound healing. The open incision has a risk of infection, dehiscence and herniation. Placement of a percutaneous endoscopic gastrostomy tube (PEG) offers the advantages of a minimally invasive technique, but relies on trans-illumination for blind placement of the finder needle. The major complication rate of PEG placement is $2 \%$ to $10 \%$, with a mortality that approximates $1 \%$ [58]. Results are mixed regarding the relative superiority of percutaneous endoscopic or radiologic techniques [6-9].

Correspondence to: Carlos E. Marroquin,, University of Vermont Medical Center, 1 South Prospect Street, Burlington, VT 05401, Tel: 802-847-4774; E-mail: Carlos.Marroquin@uvmhealth.org

Key words: $\mathrm{MeSH}$ terms, transplantation, immunosuppression, malnutrition, patient safety, deconditioned, liver

Received: March 04, 2017; Accepted: March 25, 2017; Published: March 28, 2017 
Common comorbidities of transplant patients including immunosuppression and hypoalbuminemia can dramatically increase the risks of percutaneous procedures in this population $[5,10,11]$. Since percutaneous techniques lack the ability to suture the gastric wall to the abdominal wall, early dislodgement with peritonitis is a potential surgical emergency [12] in an already compromised immunosuppressed patient. Jejunal access, which is the ideal site for delivery of nutrients, for feeding is not possible with the percutaneous technique. Gastric perforation during PEG placement in the immune-compromised patient likely carries a greater morbidity than in the immune competent as they are unable to mount an immune response and have limited ability to wall off infections. Additionally, delayed healing in this group predisposes to a lack of sinus maturation enhancing and prolonging the risk of PEG dislodgement and leak. While the risks of surgical gastrostomy placement has been traditionally regarded as higher than minimally invasive modalities, new laparoscopic techniques have received insufficient consideration $[7,13]$ and are, in our opinion, superior to blind techniques.

Since the early 1990s, descriptions of techniques in operations ranging from laparoscopic cholecystectomy to minimally invasive esophagectomy have appeared in the literature. Interestingly, there has been a paucity of interest on the placement of laparoscopic feeding tubes. In certain patient populations, particularly those with multiple barriers to adequate wound healing, the laparoscopic $G$ or J tube offers distinct advantages over both the traditional open technique and PEG. Laparoscopic placement offers the advantages of a minimally invasive technique while making it possible for direct visualization of tube placement and the ability to anchor the gastric or jejunal wall to the anterior abdominal wall. In addition to allowing access to the jejunum, it is the ability to anchor the feeding tube under direct visualization that offers a margin of safety that other techniques, including use of percutaneous fasteners, cannot offer. This approach can be performed in a timely, safe and efficient manner. For these reasons we offer the following technique for laparoscopic placement of a gastrostomy or jejunostomy feeding tube.

The patient and their families should be fully informed of the alternatives to laparoscopic technique described above and once informed consent is obtained the patient is prepared for the operating room. Two $5 \mathrm{~mm}$ and one $10 \mathrm{~mm}$ port, a general laparoscopy tray, a 5 $\mathrm{mm} 30$ degree scope, endosuture (or laparoscopic suture), endoclosure device and laparoscopic feeding tube kit are needed (Figure 1). General anesthesia is also required. The abdomen is prepared with betadine or chlorhexadine surgical prep and draped in the usual fashion. Initially, a $10 \mathrm{~mm}$ infraumbilical port is introduced and once adequate insufflation is achieved a left lateral $5 \mathrm{~mm}$ trocar is placed in the midclavicular, subcostal region at the level of T10. The third trocar $(5 \mathrm{~mm})$ is placed in the mirror image position on the right side. The camera is positioned in the left lateral trocar and any needed adhesiolysis is performed. If a gastrostomy is to be placed, the stomach is mobilized while the patient is placed in reverse Trendelenberg to deliver the small bowel and transverse colon into the lower abdomen. Alternatively, if a jejunostomy tube is desired, a loop of jejunum, 15 to $20 \mathrm{~cm}$ distal to the ligament of Treitz is identified by lifting the transverse colon up above the left lobe of the liver and stomach with the patient in Trendelenberg (Figure 2). The stomach or bowel is brought into direct apposition with the abdominal wall in left subcostal region to ensure that tension free suturing can be accomplished. This may necessitate reducing the pneumoperitoneum to 10 or $12 \mathrm{mmHg}$.

Once the ideal, tension free, intra-peritoneal position is found, the abdomen is palpated until the location on the abdominal surface is identified. This is labeled with a marking pen. Four addition sites are marked approximately $1 \mathrm{~cm}$ from the tube site at four quadrants oriented about the tube placement site. These will outline the four corners of a box with the tube site in the center (Figure 3 ). These represent the locations where the endoclosure needle will be passed into the peritoneum and used to grasp the sutures and pulled through the abdominal wall. The objective is to have the feeding tube enter the bowel with four circumferential sutures buttressing it to abdominal wall. The marked endo-closure site incisions are made approximately 2 $\mathrm{mm}$ in size and the tube site, slightly larger at about $4 \mathrm{~mm}$.

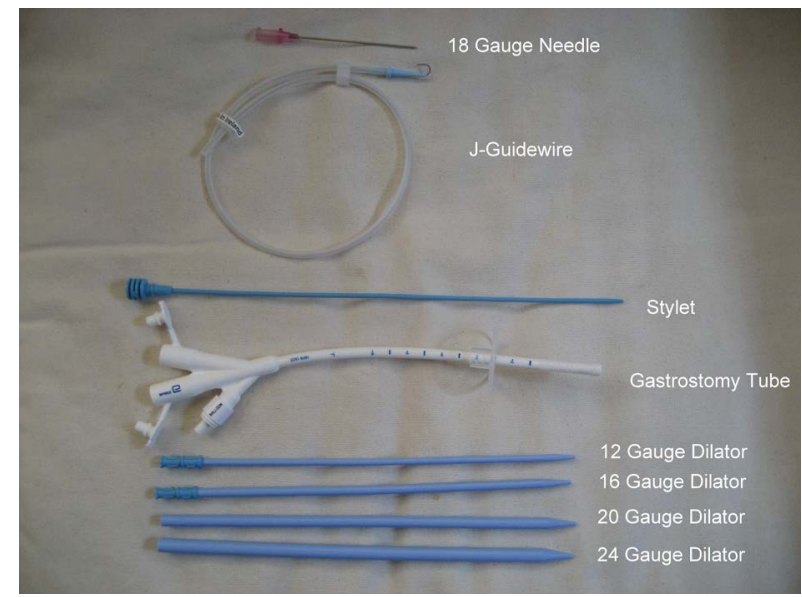

Figure 1. Needle, Dilators and Tube needed for G-tube or J-tube placement

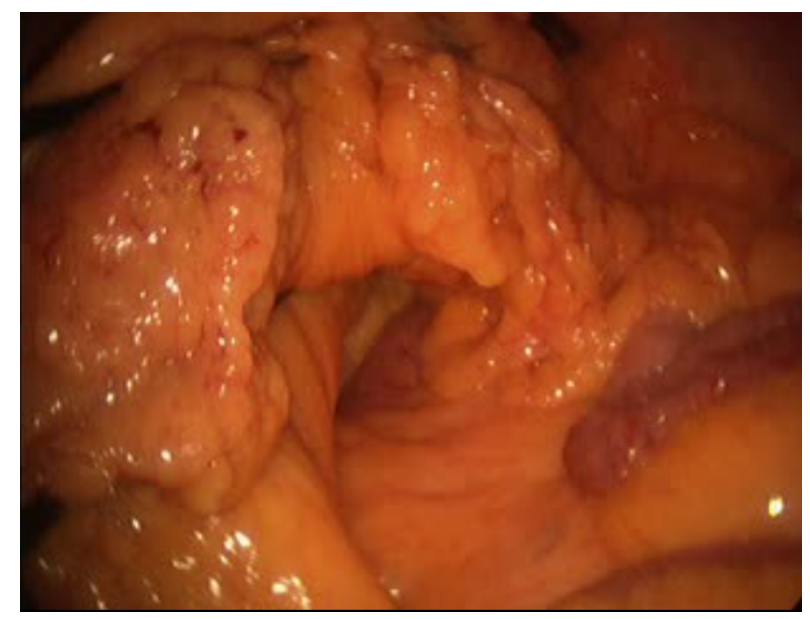

Figure 2. J tube: To identify the Ligament of Treitz, place patient in Trendelenberg position, and lift transverse colon above left lobe of the liver and stomach.

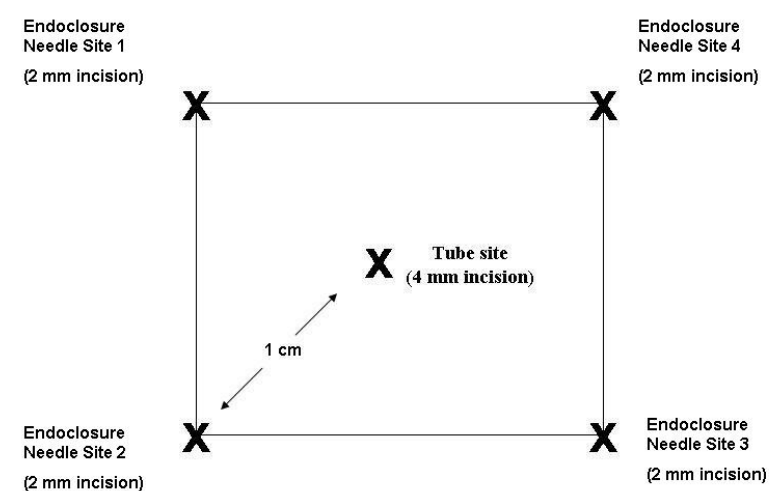

Figure 3. Placement of Tube Site and Endoclosure Needle Site Incisions 1,2, 3 and 4 for both G-tube and J- tube procedures. 
Attention is turned back to the stomach or loop of bowel. Anticipating where the tube will be inserted, three separate 2.0 nonabsorbable sutures are placed in series through the seromuscular layer $1 \mathrm{~cm}$ apart at positions 1,2 , and 3 positioned around the eventual tube entry site (Figure 4 for G-tube and 11 for J-tube). An endo-closure device is passed through the corresponding skin site and introduced into the peritoneal space. One end of the suture is pulled out of the peritoneum and the endo-closure device is reintroduced through the same incision with care to enter the peritoneum through a separate site in the fascia. This is important as this provides "purchase" on the fascia that will secure the stomach or jejunum against the abdominal wall. The other end of the suture is grasped and pulled through the abdominal wall insuring there is an adequate bridge of peritoneum and fascia to hold the suture once it is tied. They are held in place with a hemostat clamp, and are tied later. The same process is repeated with the second and third stitch. The fourth and final stitch is not placed until after the tube is inserted into the hollow viscus. If the final suture is placed before the tube is inserted, it could obstruct the view while penetrating the GI tract. When placing jejunostomy feeding tubes, placing the fourth stitch at the end also serves to help orient the tube placement downstream through the small bowel.

Once the first three stitches have been placed, the hollow viscus is lifted toward the abdominal wall. Placing the tube is performed using a modified Seldinger technique. The finder needle is passed through the central incision site, through the abdominal wall and into the stomach or jejunum under direct visualization. This should be done such that the needle enters the center of the previously placed sutures (Figure 5). Slight tension should be placed on the sutures to permit easy penetration of the needle into the stomach or jejunum. With the tip of the needle in the lumen, the stomach or bowel is briefly inspected to ensure the needle has not passed through the opposite side. At this point, a guidewire is passed though the needle (Figure 6). This should be done without any resistance. Any resistance may be suggestive of incomplete passage of the needle and dissection through the layers of the bowel wall. The guidewire is advanced and the needle removed. A series of dilators are subsequently passed through the abdominal wall dilating the tract over the guidewire and removed (Figure 7). When placing a G-tube, the tube with its internal dilator are placed through the dilated tract into the stomach. The balloon is inflated and the guidewire and dilator are removed. When placing a jejunostomy tube, the peel-away sheath is placed over the guidewire and once again advanced over the guidewire into the jejunum. Once in place,

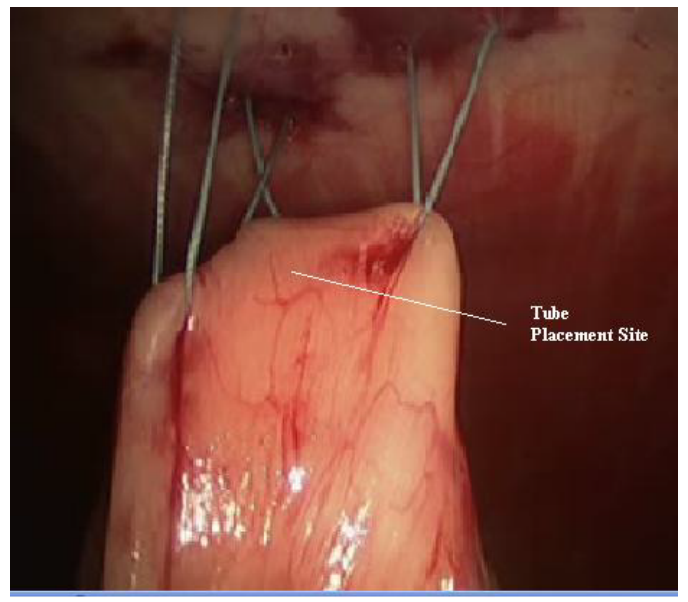

Figure 4. G tube: Placement of Stitches 1,2 and 3.

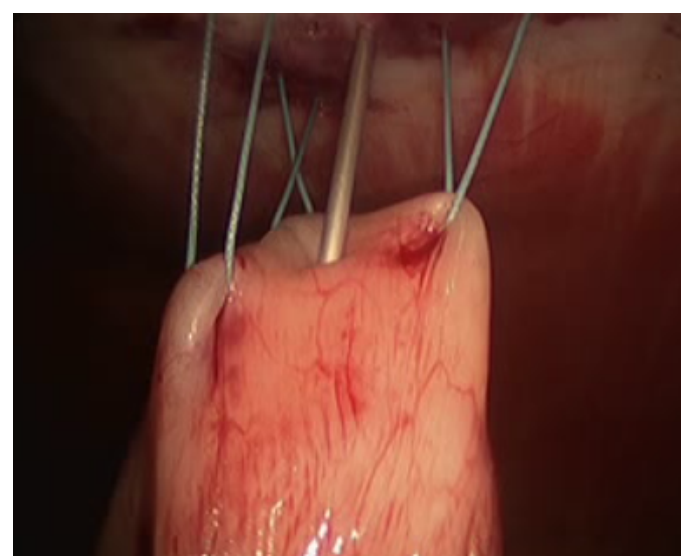

Figure 5.G tube: Place 18 gauge needle in desired tube placement site, in the center of stitches 1,2 and 3 .

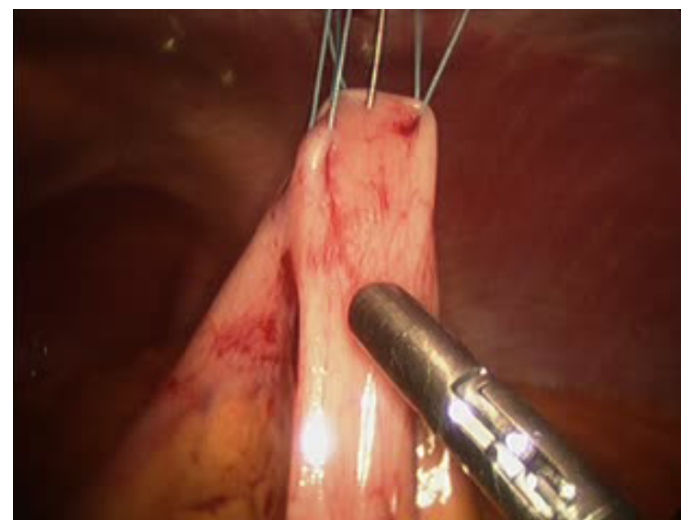

Figure 6. G tube: Only remove needle after advancing J-guidewire (guidewire shown here) using Seldinger technique.

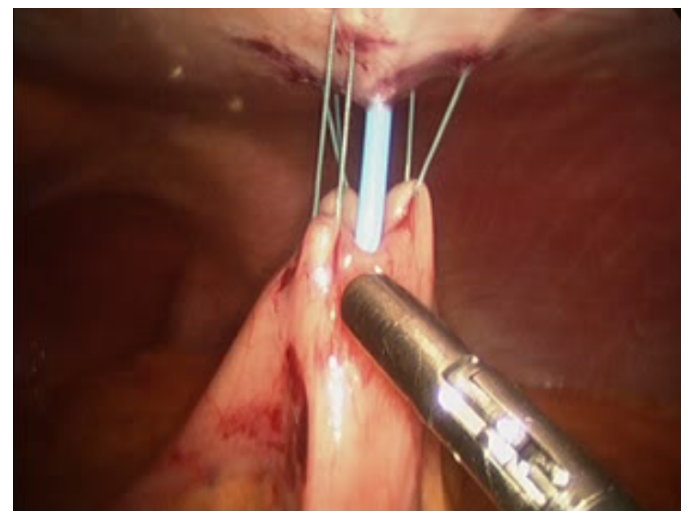

Figure 7. G tube: Use $12 \mathrm{G}, 16 \mathrm{G}, 20 \mathrm{G}$ and $24 \mathrm{G}$ dilators to subsequently dilate site of entry of feeding tube.

the guidewire is removed, leaving the peel-away sheath in place. The feeding tube may be threaded through the sheath and into position. The sheath is peeled away. With the tube in place, the 30-degree scope is positioned to examine all sides of the bowel to again ensure that the tube has not been penetrated the bowel/stomach and that it does not appear to be dissecting the layers of the bowel/stomach.

With the tube successfully positioned, the fourth suture can be placed in the fourth position such that the tube is circumferentially buttressed with suture (Figure 8). The ends of all four sutures are extracorporeal and can be tied and secured. The bowel is inspected to 
ensure that that it is snug against the abdominal wall, but not so tight to cause necrosis (Figure 9). If a jejunostomy tube was placed, two or three additional securing sutures are placed downstream and fastened with the endoclosure device approximately 3 and $5 \mathrm{~cm}$ distal to the tube site (Figures 10-14). This is done to reduce the likelihood of volvulus around a fixed point. Before closure, the tube is flushed with methylene blue to evaluate for any leaks. Leaks are likely related to failure of one or more of the circumferential sutures to adequately buttress the tube entry site, bowel perforation during needle penetration or during the process of dilation. In our opinion, this is a critical step as these can easily be repaired if recognized and prevents a serious complication we are trying to avoid.

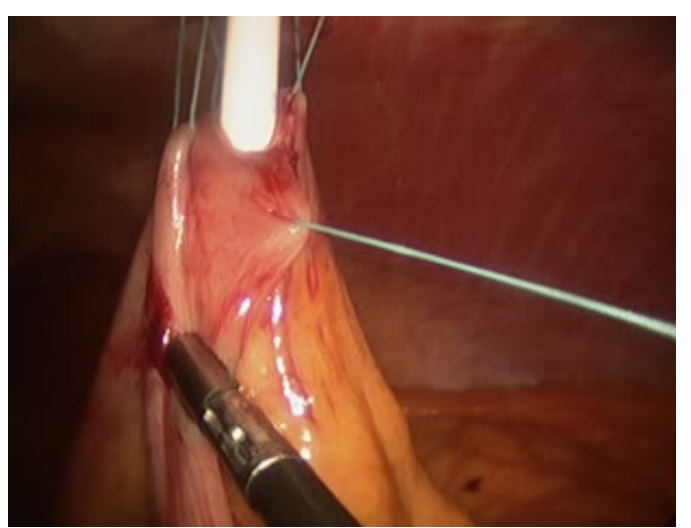

Figure 8. G tube: Placement of $4^{\text {th }}$ Buttressing Suture only after feeding tube has been successfully position.

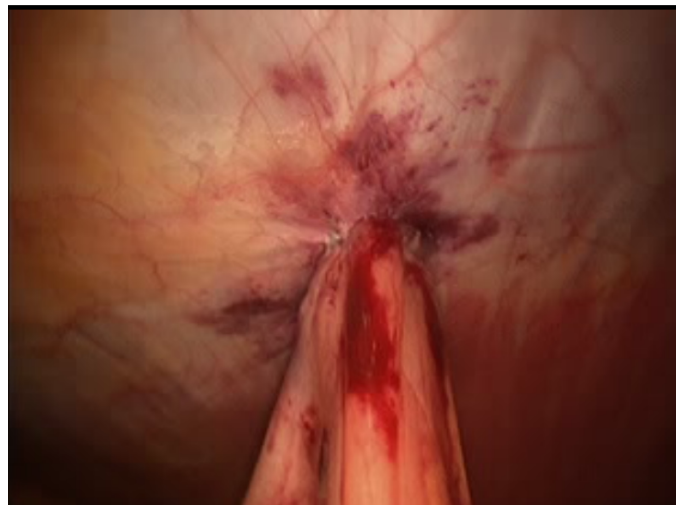

Figure 9. G tube: final placement of all 4 buttressing sutures.

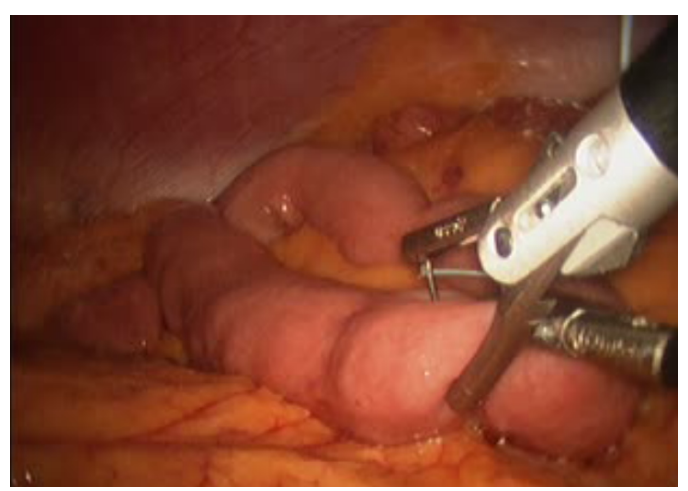

Figure 10. For $\mathrm{J}$ tube procedure, place initial stitch in loop of bowel $20-30 \mathrm{~cm}$ distal to Ligament of Treitz.

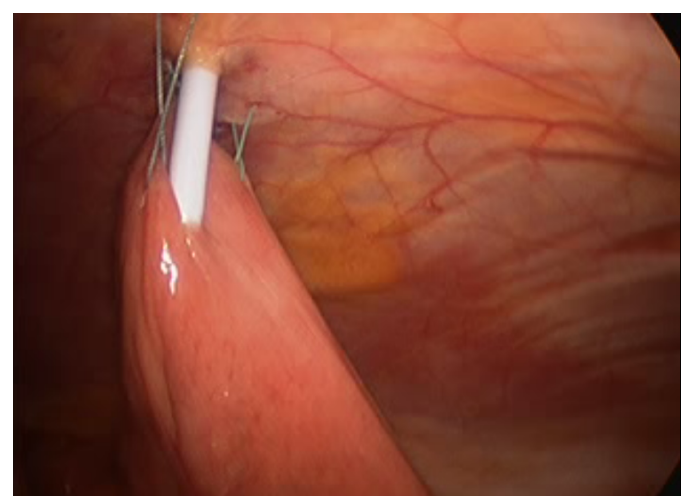

Figure 11. J tube: Placement of Stitches 1,2 and 3.

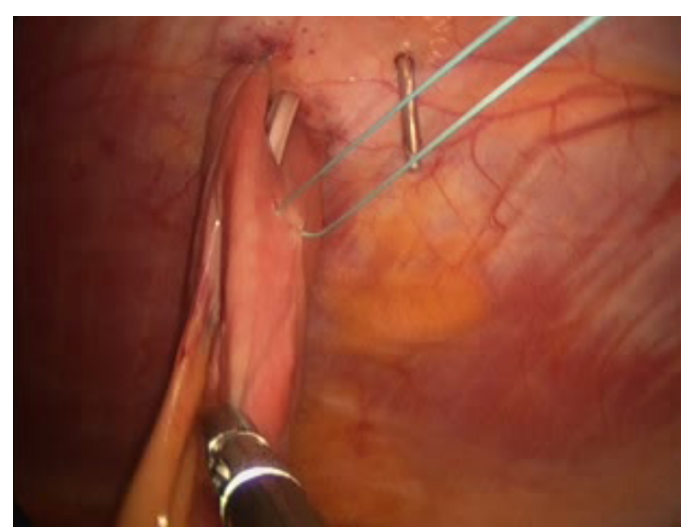

Figure 12. J tube:Placement of $4^{\text {th }}$ stitch only once tube is in place.

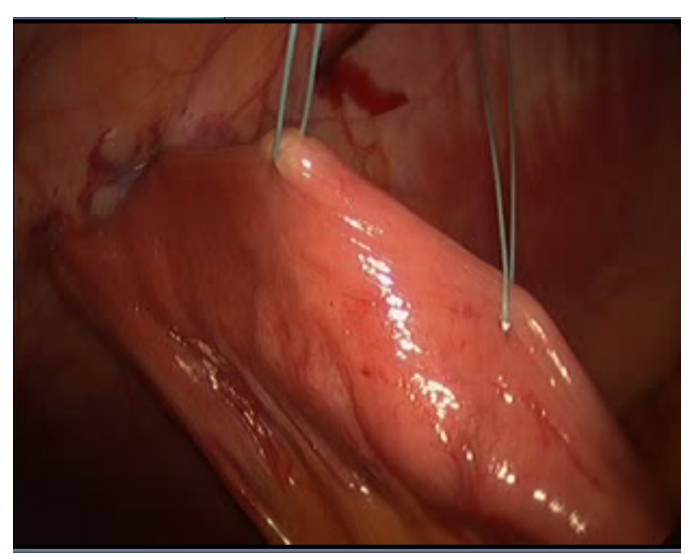

Figure 13. $\mathrm{J}$ tube: Placement of securing sutures $3 \mathrm{~cm}$ and $5 \mathrm{~cm}$ distal to $\mathrm{J}$ tube site to prevent torsion.

Finally, the tube is sutured to the skin. We prefer to suture both the tube to the skin, as well as the phalange to the skin. This helps to prevent tube migration and dislodgement. The abdomen is inspected for hemostasis and the incisions closed after the ports are removed. The tube may be capped or put to gravity for 24 hours. Normal saline infusion is begun on POD1 and the flow rate is increased over four hours. We use normal saline to assess the mechanical flow. When and if complications occur, they are evident early in the postoperative course. Detecting problems with normal saline results in less morbid complications than formulary feeds. If the patient tolerates normal saline infusion, trickle feeding may begin and advanced as tolerated toward goal. 


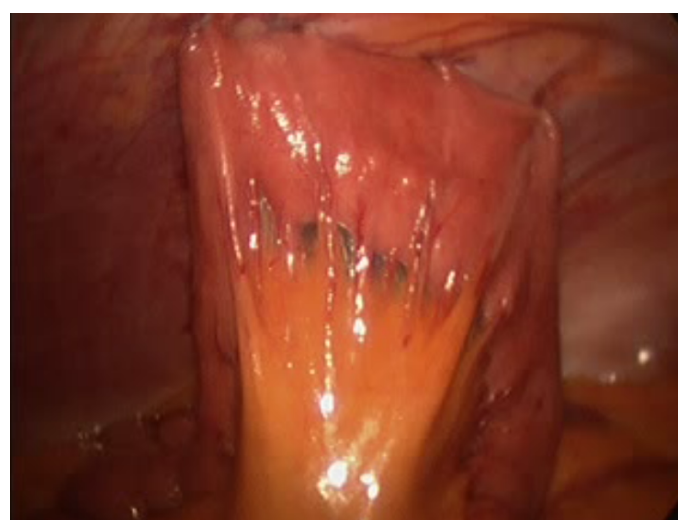

Figure 14. $\mathrm{J}$ tube placement with placement of all 4 buttressing sutures around $\mathrm{J}$ tube and distal securing sutures at $3 \mathrm{~cm}$ and $5 \mathrm{~cm}$ distal to $\mathrm{J}$ tube site.

Although this technique does not eliminate all of the risks associated with operating on malnourished, immunosuppressed patients, it does mitigate some of the complications that may be encountered with previously used blind, percutaneous techniques while providing the necessary enteral access malnourished patients require.

\section{Disclosure}

The authors report no proprietary or commercial interest in any product mentioned or concept discussed in this article.

\section{Author contributions}

Eric Hansen: Co-author of majority of manuscript text, major contributor to study design. Conor O'Neill: Assisted with manuscript design and figures/imaging. Provided manuscript revision and editing. Carlos E. Marroquin: Co-author of majority of manuscript text, major contributor to study design. Provided the final revisions and additions to the manuscript.

\section{References}

1. Kudsk KA (2002) Current aspects of mucosal immunology and its influence by nutrition. Am J Surg 183: 390-398. [Crossref]

2. Mainous MR, Deitch EA (1994) Nutrition and infection. Surg Clin North Am 74: 659 676. [Crossref]

3. Koretz RL, Avenell A, Lipman TO (2007) "Does enteral nutrition affect clinical outcome? A systematic review of the randomized trials." Am J Gastroenterol 102: 412429. [Crossref]

4. Montecalvo MA, Steger KA, Farber HW, Smith BF, Dennis RC, et al. (1992) Nutritional outcome and pneumonia in critical care patients randomized to gastric versus jejunal tube feedings. The Critical Care Research Team. Crit Care Med 20: 1377-1387. [Crossref]

5. Blomberg J, Lagergren P, Martin L, Mattsson F, Lagergren J (2011) Albumin and C-reactive protein levels predict a short-term mortality after percutaneous endoscopic gastrostomy in a prospective cohort study. Gastrointest Endosc 73: 29-36. [Crossref]

6. Ho SG, Marchinkow LO, Legiehn GM, Munk PL, Lee MJ (2001) Radiological percutaneous gastrostomy. Clin Radiol 56: 902-910. [Crossref]

7. Silas AM, Pearce LF, Lestina LS (2005) Percutaneous radiologic gastrostomy versus percutaneous endoscopic gastrostomy: A comparison of indications, complications, and outcomes in 370 patients. Eur J Radiol 56: 84-90. [Crossref]

8. Wollman B, D'Agostino HB, Walus-Wigle JR, Easter DW, Beale A (1995) Radiologic, endoscopic, and surgical gastrostomy: an institutional evaluation and meta-analysis of the literature. Radiology 197: 699-704. [Crossref]

9. Leeds JS, McAlindon ME, Grant J (2010) Survival analysis after gastrostomy: a single-centre, observational study comparing radiological and endoscopic insertion. Gastroenterol Hepatol 22: 591-596. [Crossref]

10. Tominaga N, Shimoda R, Iwakiri R, Tsuruoka N, Sakata Y, et al. (2010) Low serum albumin level is risk factor for patients with percutaneous endoscopic gastrostomy. Intern Med 49: 2283-2288. [Crossref]

11. Baltz JG, Argo CK, Al-Osaimi AMS, Northrup PG (2010) Mortality after percutaneous endoscopic gastrostomy in patients with cirrhosis: a case series. Gastrointest Endosc 72: 1072-1075. [Crossref]

12. Schulenberg E, Schule S, Lehnert T (2010) "Emergency surgery for complications related to percutaneous endoscopic gastrostomy." Endoscopy 49: 872-874. [Crossref]

13. Joseph M, Myers MO (2010) Laparoscopic-assisted percutaneous gastrostomy tube placement in the initial management of resectable esophageal and gastroesophageal junction carcinoma. J Am Coll Surg 211: e21-e24. [Crossref]

Copyright: (C2017 Hansen E. This is an open-access article distributed under the terms of the Creative Commons Attribution License, which permits unrestricted use, distribution, and reproduction in any medium, provided the original author and source are credited. 